\title{
A Double Stage DC/DC Converter for LED Lighting Automotive Systems
}

\author{
Marco Failla ${ }^{1}$, Gianpaolo Vitale ${ }^{2}$ \\ ${ }^{1}$ Dipartimento di Energia, Ingegneria dell'Informazione e Modelli Matematici, Università \\ degli Studi di Palermo, 90128 Palermo, Italy \{marco89.failla (at) gmail.com\} \\ ${ }^{2}$ ICAR, Institute for high performance computing and networking, National Research Council (CNR), \\ Via Ugo La Malfa, 153, 90146 Palermo \{gianpaolo.vitale (at) icar.cnr.it\}
}

\begin{abstract}
In this paper a DC/DC converter, conceived to be employed for LED lighting in automotive application, is proposed. It is composed of two stages to fit both to the constraint to be powered by a variable supply voltage and to deliver a constant output load current with reduced ripple to supply the LED.

Firstly the LED has been experimentally characterized to obtain a model to be implemented on a circuit simulator. Then the design of the converter has been carried out, including the control laws, to verify the compliance with the constraints. Finally, a circuit based on commercial devices is proposed to be applied both to lighting and to direction indicators light, including current and temperature protection. Results, given in simulation, show good performance achievable with a low-cost hardware.
\end{abstract}

\section{Key words}

LED, automotive, lighting, efficiency, DC/DC converter, current control, white LED, current ripple.

\section{Introduction}

This work arises from the proven and frequent need to equip the vehicles with LED lighting systems, efficient, compact and characterized by intense light flows [1-5]. The front lighting systems must allow safe and comfortable driving in poor visibility conditions or in adverse weather conditions. In the past the classical solutions were based on "filament lamps" in which light is produced by means of a threadlike conductor which is heated to incandescence by the passage of an electric current; nowadays LED based Lighting systems have been assuring high luminous flux maintaining high efficiency. The use of LED is already recognized as a way to save energy reducing the $\mathrm{CO}_{2}$ emissions [6], for this reason, the design of LED based systems is acquiring more interest. On the other hand, the LED supply is still a challenge in engineering world and among the solutions proposed in the literature, the state of the art is achieved with suitable
DC/DC converters [7-13].

The DC/DC converter is the best choice in terms of efficiency and reliability, but the need to be very compact and often integrated into the lighting module itself, in particular for automotive applications, makes its design and its circuit layout more complex.

The most used non-isolated DC/DC converter topologies are: the Buck or step-down converter, Boost or step-up and Buck-Boost. Often such topologies are not sufficient to achieve the desired results and a more complex topology has to be devised. It must take into account also that the automotive environment gives a supply voltage whose value can vary in a wide range and that it requires a high reliability for safety driving conditions.

The goal of this circuit consists on supplying a LED with constant current, very low ripple and limited size. The work was mainly focused in the architecture of the converter, to identify a circuit diagram capable to meet the requirements imposed by the application, and to simplify the control as much as possible, reducing both in number and in size all the passive components. Two main constraints should be considered:

- Ripple of voltage or current around mean value. This is an unwanted phenomenon, linked to the switching of the power supply. The current ripple must be less than $5-6 \%$ of the nominal current value, otherwise the human eye would notice variations in light intensity.

- LED model for simulation: a model for a LED is an equivalent circuit or a function able to approximate the behavior of the device on the basis of the information provided by the datasheet or by experimental measurements. The LED model must allow accurate simulation of the overall behavior of the converter and is therefore of crucial importance. 


\section{LEDs: features and driving requirements}

A LED is a semiconductor device that allows direct energy conversion from electric energy into light energy. It is formed by a $\mathrm{p}-\mathrm{n}$ junction, obtained ideally by approaching a n-type semiconductor to a p-type one. Under forward voltage the height of the potential barrier across the junction is reduced, so the electrons can cross the junction where they recombine with holes. The recombination process releases energy in the form of visible light, at a wavelength given by (1):

$\lambda=\frac{h c}{E_{g}}$

where $E_{g}$ is the energy gap, $h$ is the Planck constant, $c$ is the light velocity. The energy gap is a feature of the material and the emitted light depends on it.

On the other hand, the white, being a wide band source, is the only color that allows a natural illumination of the environment to pursue safety driving condition, unfortunately, in nature, a material that emits white light does not exist yet.

White LEDs, suitable for automotive lighting applications, are made with a blue LED and a phosphor that absorbs the blue light and emits to higher wavelenght resulting in a light spectrum (figure 1) perceived as white light by the human eye.

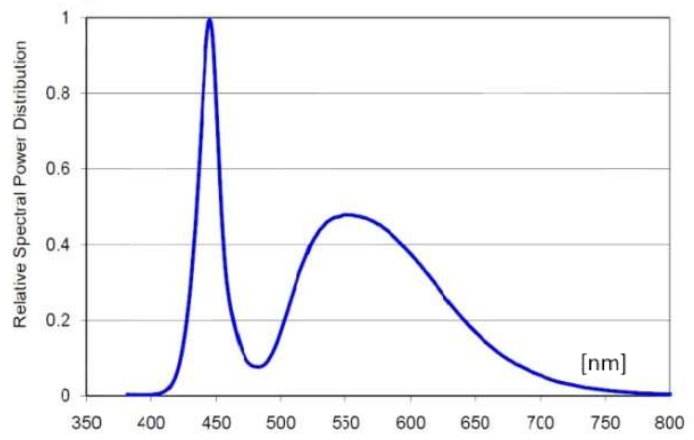

Fig.1: white LED spectrum

White power LEDs can reach efficiency between 25 and $100 \mathrm{~lm} / \mathrm{W}$, higher than most common incandescent or halogen lamps and therefore can be used for automotive lighting applications, including daytime light, fog light, high beam and low beam. Unlike traditional sources, LEDs offer the following advantages:

- Long lifetime

- Power saving

- Reliability

- Low power dissipation

- Dimming capability

- Compact size

Anyway LEDs are semiconductor devices and are very different from traditional light sources so they require proper drivers to adjust the current. The main challenge in the development of LED drivers are directly related to the photometric, electrical and thermal characteristics of the device, briefly described below:

1. Photometric characteristics: there is a strong linear dependence between current and luminous flux emitted, so to maintain constant the flux, the driving current must be equally stable.

2. Electrical characteristics: the LED has a nonlinear (exponential) I- $V$ characteristic. When the driving voltage exceeds the threshold voltage of the device, it starts to emit light, however small variations in the voltage cause large variations in current (and flux) due to the strong slope of the characteristic above the threshold.

3. Thermal characteristics: for a given voltage, an increase in the junction temperature leads to an unwanted increase in current and to a further increase in temperature. A degenerative process is triggered.

There are many drivers able to supply a LED with constant current but DC/DC converters are the best choice.

The DC/DC converter has to be controlled, with techniques that will be analyzed below, to react quickly to variations in supply voltage and thus to ensure a constant driving current, a luminous flux that is stable over time and insensitivity to the variability of external conditions, including the temperature. The control variable is the duty cycle or the switching frequency of the converter.

\section{LED modelling}

A LED is a $p-n$ junction diode whose characteristic exhibits a strong non-linearity (figure 2).

For voltage values lower than the threshold voltage $V_{\gamma}$, the diode does not conduct and therefore it does not emit light. As soon as the voltage exceeds the threshold the diode starts to conduct and light is emitted.

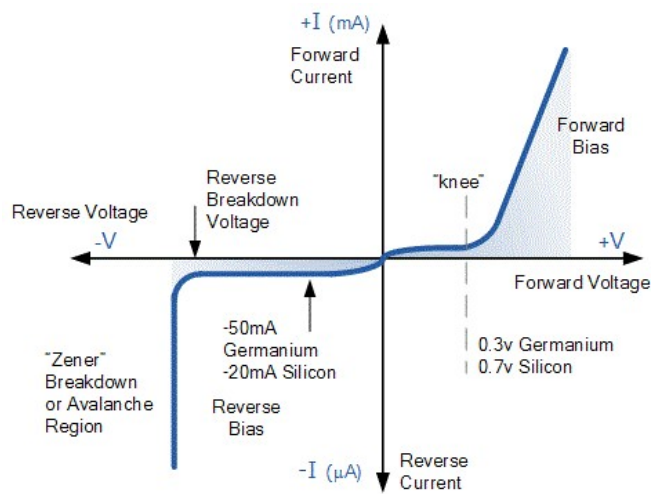

Fig. 2: LED I-V characteristic

The current-voltage characteristic shown in figure 2 is described by the Shockley equation (2):

$I_{D}=I_{S}\left(e^{\frac{q V_{D}}{n k T}}-1\right)$

where $I_{D}$ is the current flowing through the diode, $I_{S}$ is the inverse saturation current, $V_{D}$ is the voltage applied at the terminals of the diode, $q$ is the charge of the electron, $k$ is the Boltzmann constant, $T$ is the junction temperature expressed in kelvin, $n$ is the ideality factor of the diode.

Because of this feature, which exhibits a high slope 
section, it is necessary to identify a model able to correctly represent the behavior of the diode around the chosen working point.

The circuit representing a piecewise linear model, based on the exponential characteristic of the diode, is shown in figure 3.

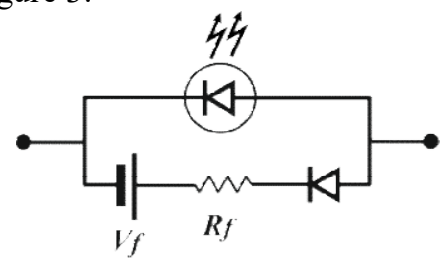

Fig. 3: piecewise model equivalent circuit

The model consists of an ideal voltage generator ? which represents the threshold voltage of the LED, in series with an ideal diode and a resistance ? which takes into account the dynamic resistance of the LED. This resistance is equivalent to the inverse of the slope of the curve at a given work point.

In many cases, the power LED manufacturer gives only the graphic feature of the device in the data-sheet without any mention of the function that describes it. Nevertheless, even the characteristic parameters of the device, such as the inverse saturation current or the ideality factor, are missing and the Shockley equation cannot be used to model the LED. However, an alternative approach can be followed, described briefly below.

From the current-voltage graph the coordinates of some points in the curve are obtained, in number proportional to the degree of accuracy desired to mathematically describe this curve.

Infinite curves pass through the selected points, but the characteristic of the LED cannot be so different from the ideal one defined by Shockley. The problem leads back to determine an exponential or polynomial function (at most of the second order) suitable to fit the chosen points.

There are many softwares able to do that, so that, once this function is obtained, it can be implemented directly on the simulation software thanks to a non-linear element supplied with the library. The load will therefore consist of a functional block $I=f(V)$ that perfectly simulates the behavior of the LED. This model is known as Polynomial.

The LED chosen is a COB LED, it is formed of a multiple LED chips (more than 9 in general) welded by the manufacturer in a substrate, often ceramic/metallic, to form a single module, with two external terminals.

The most common COB LEDs are the Cree Xlamp ones. For the experimental phase the Cree CXA1304 LED was chosen, easy to find and with an excellent cost/performance ratio, it is available with 9, 18 and 37 $\mathrm{V}$ rated voltage with a maximum current of $1,0.5$ and
0.25 A respectively.

Current and voltage measurements have been carried out to verify their compatibility with the values provided by the datasheet. The test bench consists of a heatsink, a Buck converter with LM2956S controller, a commercial $12 \mathrm{~V}$ power supply and a multimeter. The use of the heatsink has become mandatory after the first phase of measurements because the temperature reached by the LED case has exceeded the limits suggested. The measured values are shown in figure 4, together with a picture of the test rig.

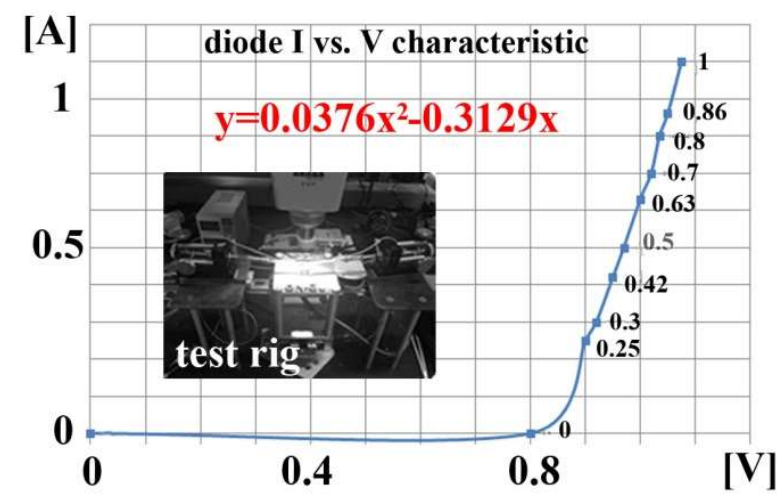

Fig. 4: polynomial interpolation of measured values

The LED showed a behavior in line with expectations with negligible variations from the values extracted from the manufacturer's data-sheet.

By computational software, a classic polynomial fitting, whose trend is shown in figure 4, is performed and the approximate second order function has been obtained:

$I_{o}=0,0376 V_{o}^{2}-0,3129 V_{o}$

\section{Designed DC/DC converter}

The electric scheme of the converter is shown in figure 5. This is a double stage with an input Buck and interleaved Boost in the output stage. Both converters are synchronous. The choice of a double conversion stage allows the variation of the LED power supply as the battery voltage changes to be minimized.

The input of the Buck is connected to a square wave generator that supplies a voltage in the range 10.5-14.5 $\mathrm{V}$ to simulate the battery status under different loads and in different vehicle operating conditions.

The output current of the Buck is controlled by hysteresis. The current reference is chosen according to the current required by the load which must be $0.7 \mathrm{~A}$ to get the required luminous flux.

Since the second stage sensing is performed at the input of the converter itself, the required current does not coincide with that of the load but is multiplied by a factor that depends on the duty cycle. 


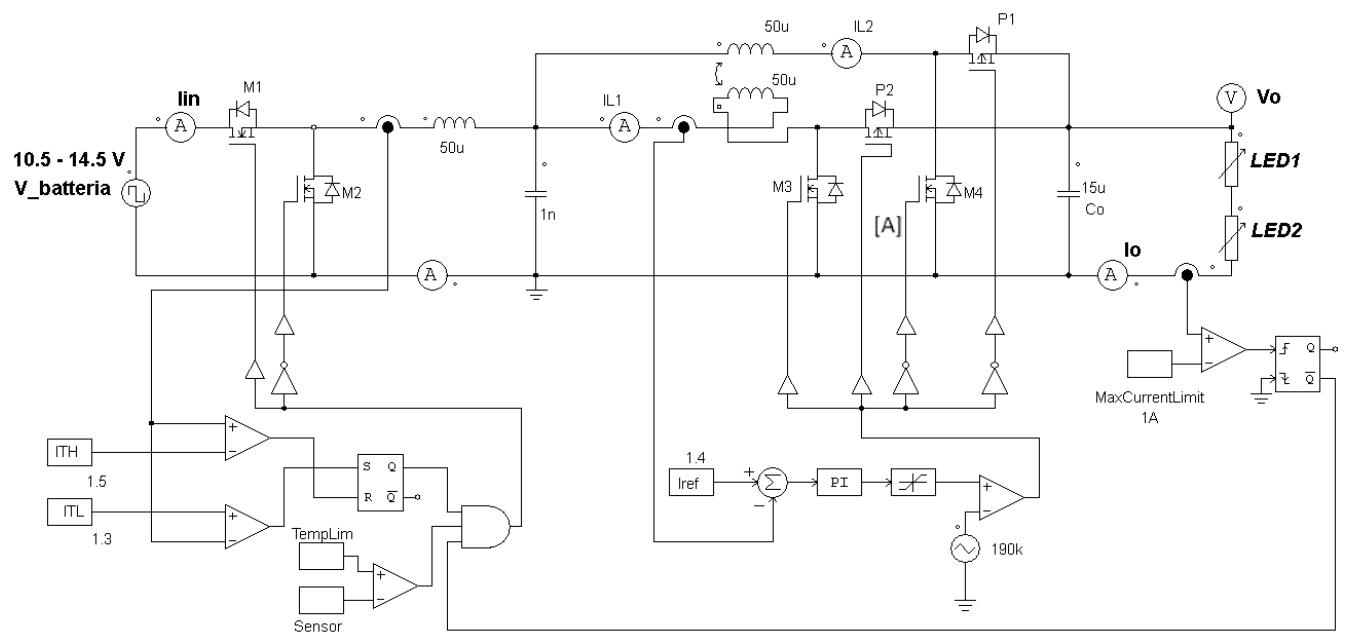

Fig. 5: Electric scheme of the designed converter

This current is given by:

$I_{S}=\frac{V_{o} I_{o}}{V_{S}}=20,4 \cdot \frac{0,7}{10,2}=1,4 \mathrm{~A}$

In (4), the voltage $V o$ and output current $I o$ are the design constraints. Instead, the intermediate voltage $V_{s}$ must be chosen appropriately. In fact, the current control of both the Buck and the Boost converter should not place constraints on the intermediate voltage, however, the control is based on a concept that does not distort the circuit architecture and is also the main contribution to this work. It places a stringent constraint on the intermediate voltage, which must be about half of the desired output voltage.

This current, that flows in the input inductor, represents the control input for the hysteresis comparator which keeps constant the current on the branch, acting on the status of the power switch.

The output Boost is configured as a classic interleaved topology that nonetheless behaves similarly to a stacked topology, although there is no filtering capacitor for the DC component (figure 6) on the secondary branch.

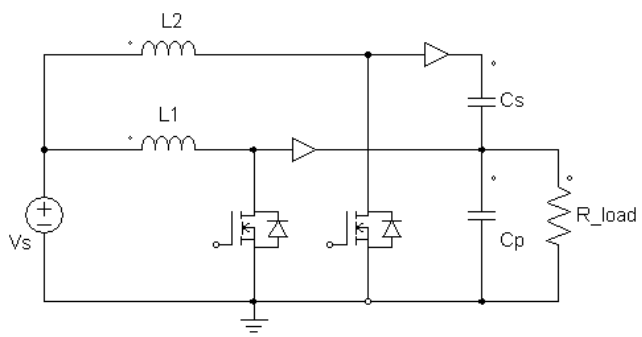

Fig. 6: classic stacked topology

A similar result was obtained thanks to a coherent control design, carried out only on the primary branch of the converter: the current drawn by the output stage inductor is compared with a reference value that coincides with the entire DC current delivered by the primary stage, therefore, the whole continuous component required by the load flows on the main branch, instead the average current on the other branch is null (figure 7). The output stage behaves as a stacked Boost with all the related advantages. In particular, since the duty cycle is not imposed externally, the cancellation of the ripple takes place anyway, whatever its the value.

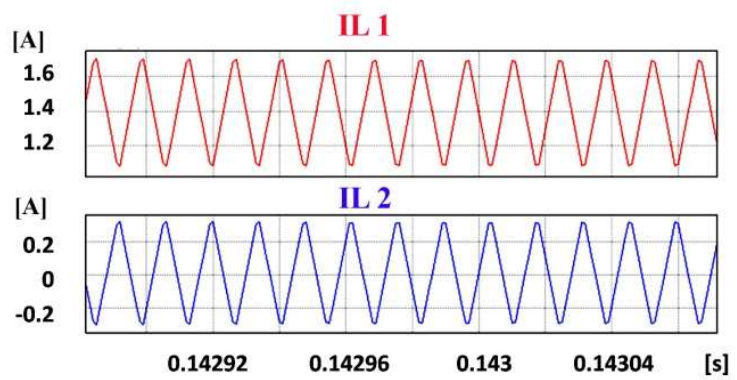

Fig. 7: currents in a stacked like Boost: current in the main inductor (top) and current in the secondary inductor lacking of the DC value (bottom).

Other advantages are the increase of efficiency, the improvement of the transient and the reliability since the converter is able to work even if some components on one single branch were damaged. The benefit in terms of performance comes at the cost of one additional inductor, switch, and diode.

The output stage is current controlled by a PI regulator. The current on the primary inductor is subtracted from the reference value of $1.4 \mathrm{~A}$, the error is processed by the PI regulator and the resulting voltage is limited in order to fall within the range of use of the PWM comparator, whose inverting input is a triangular wave with a frequency of $190 \mathrm{kHz}$. The comparator drives the power of one of the two branches with a duty cycle value (D) and the switch of the other branch with the complementary value (1-D). The value of the output capacity is chosen on the basis of the simulations, to obtain a ripple current less than or equal to $40 \mathrm{~mA}$.

Additional functions have been provided to prevent possible damages in critical operating situations such as an output short circuit or an undesired or uncontrolled increase in temperature.

A protection mode called "Hiccup Mode" has been implemented against short circuits: if the current exceeds the maximum value, a comparator activates an edge triggered monostable, which generates a pulse that turns off the switch of the input stage for a time 
equal to 10 times the switching period. In this case, the Buck operates with the input disconnected from the battery and the output capacity is discharged on the secondary stage, progressively reducing the current. Once the impulse is over, normal operation is reactivated with the current returning to normal operation, but if the malfunction condition is still present, another protection cycle starts, and so on until the anomalous condition is no longer present.

The control also provides a shutdown protection of the converter in case of LED overheating. A sensor responsible for measuring the temperature sends the information to a comparator whose threshold is set to the maximum value suggested by the manufacturer and indicated in the data-sheet. When the temperature exceeds the threshold, the comparator triggers a low logic value and turns off the input transistor, disconnecting the power supply for as long as necessary for cooling. In this condition, the LED is off and the safety of the motor vehicle and passengers could be compromised but this condition can be recognized in advance giving a warning signal. It is therefore evident the necessity to provide the converter and the LEDs with a suitable heatsink.

\section{Realization with commercial devices}

The proposed circuit scheme is firstly analysed with ideal elements, useful for highlighting the general behavior at the first level of approximation but which in fact do not have an electronic meaning. Often they are mathematical blocks such as constants, adders and so on and if they are existing components (transistors, diodes, resistances, etc.) their behavior is absolutely ideal.

Even the control techniques, although particularly effective, use predefined blocks for which it is appropriate to find an equivalent circuit achievable with discrete, commercially available, components.

The designed converter, ready for the practical realization is shown in figure 8. All commercial components adopted are shown in Table I.

The current sensing on the inductors, by design choice, is carried out by a shunt resistance and a differential amplifier. The resistor is connected in series to the inductor and its terminals branch give two connections that reach the differential amplifier. Rather than using a discrete operational, to limit the final dimensions, one of the many integrated circuits available on the market has been chosen, in particular the Texas Instruments INA210-Q1 model. It is an integrated device that is self-powered and accepts supply voltages from $2.7 \mathrm{~V}$ up to $26 \mathrm{~V}$, it can work both in common mode and in differential mode, has a gain of $200 \mathrm{~V} / \mathrm{V}$, it is certified for use in the automotive sector with temperatures between $-40 /+125^{\circ} \mathrm{C}$, is extremely accurate $( \pm 100$ $\mu \mathrm{V}$ ), ensures a very low drift of current and has very small dimensions: $2 \times 1.25 \mathrm{~mm}$.

The sensing resistors, for measuring the current, are in series with the input inductor and to one of the two coupled inductors. The differential voltage is sent to the integrator whose output constitutes the current signal for the hysteresis controller On-Semiconductor
NCL30161. It is a constant and tunable current controller, ideal for lighting applications, operates with a variable supply voltage from 6.3 to $40 \mathrm{~V}$ and also allows dimming of the light intensity via external PWM signal. It offers thermal and short circuit protection and can achieve $100 \%$ duty cycle values. All in a very small package of $3 \times 3 \mathrm{~mm}$.

Table I: commercial components

\begin{tabular}{|c|c|}
\hline \multicolumn{2}{|c|}{ Table I: commercial components } \\
\hline Components of the converter \\
\hline On-semiconductor NCL30161 & $\begin{array}{c}\text { Hysteresis current } \\
\text { controller }\end{array}$ \\
\hline Texas Instruments INA210-Q1 & Current sensor \\
\hline Cree XLamp CXA1304 & LED \\
\hline Texas Instruments CSD18510Q5B & N-MOS \\
\hline Texas Instruments CSD25404Q3 & P-MOS \\
\hline $50 \mu H$ Coilcraft MSD1583-104KE & Coupled inductors \\
\hline $50 \mu H$ Coilcraft MSS1260T-104ML & Inductor $\boldsymbol{L}_{\boldsymbol{i n}}$ \\
\hline $15 \mu \mathrm{F}$ & Capacitor $\boldsymbol{C}_{\boldsymbol{o}}$ \\
\hline
\end{tabular}

The main difference with the scheme already seen is in the controller of the second stage, no longer PWM type with proportional regulation and integrator, but also with hysteresis. In particular, the same integrated device from On-Semiconductor was used.

The input stage capacitor has been removed, improving the reliability of the system, because it is a superfluous element in the circuit dynamics. The stability of the voltage on that node does not depend on the presence of the capacitor, so with the current control on the two stages, all the current required by the output stage is supplied directly by the inductor and does not need to be filtered.

About the output capacitor, the entire circuit architecture allowed to limit its value to only $15 \mu \mathrm{F}$, this is an excellent result despite the considered stringent specifications on the current and on voltage values. A similar capacitor, which is usually the bulkiest element, can be integrated into SMD form, occupying a very small area. The surface is therefore largely occupied by the inductors, on which there is no margin of integration in this application.

\section{Efficiency evaluation}

The ideal efficiency of a DC/DC converter is defined by the ratio between output power and input power:

$\eta=\frac{P_{o}}{P_{\text {in }}}$

where $P_{o}$ is the output power and $P_{\text {in }}$ is the input power, both calculated as a product between the average current and voltage values.

In real cases, several loss factors come into play, the most important of which are listed below:

- Conduction losses in the power switches, defined by the relation $P_{M o s}=I^{2} R_{O N}$, where $I$ is the mean or effective current value in the MOS and $R_{O N}$ is the resistance that the transistor shows 
during conduction.

- Conduction losses in diodes, linear with current, defined by the relation $P_{\text {diode }}=V_{\gamma} I$.

- Losses in the inductors, due to both the switching frequency and the parasitic resistance. This last loss factor is dominant and is described by the relation $P_{L}=I^{2} R_{\text {leak }}$.

The input power $P_{\text {in }}$ can be expressed as the sum of the output power plus a part of the power that is lost during the conversion process $\left(P_{\text {lost }}\right)$ and is given by the sum of all the contributions of interest:

$P_{\text {in }}=P_{o}+P_{\text {lost }}$

The efficiency of a converter, taking into account the losses, is given by:

$\eta=\frac{P_{o}}{P_{\text {lost }}+P_{o}}$

For a first estimation of the efficiency, the available commercial components have been taken into account. The selected MOS is a Texas Instruments N-Channel (CSD18510Q5B) of $40 \mathrm{~V}$ drain-source voltage and 100 A continuous drain current. An important feature is the resistance during conduction, which in the worst case is $1.2 \mathrm{~m} \Omega$. These specifications fully meet the requirements set by the converter, moreover, the package is quite small, $5 \times 6 \mathrm{~mm}$, ideal to integrate the electronics in a single module. The P-MOS considered is a transistor of the same family (CSD25404Q3) and the same size, $20 \mathrm{~V}$ drain-source voltage and $60 \mathrm{~A}$ direct current. The conduction resistance is higher, in the worst case $10.1 \mathrm{~m} \Omega$.

With reference to the circuit diagram, the total power dissipated by the MOS is the sum of the individual powers. The total power lost in the transistors present in the converter results:

$P_{\text {Mos }}=13,82 \mathrm{~mW}$

The coupled inductors can be easily found on the market and are usually enclosed in a plastic package of variable dimensions based on the desired inductance value. Coilcraft's MSD1583 series lends itself well to DC/DC conversion applications where high currents, low losses, and high switching frequencies are required. The $50 \mu \mathrm{H}$ version has a package of $15 \times 15 \times 8$ $\mathrm{mm}$ and is tested for a continuous current of $1.75 \mathrm{~A}$, higher than the value imposed by the circuit which stands at $1.4 \mathrm{~A}$. The parameter of interest in the calculation of losses is the parasitic resistance which in the selected model (MSD1583-104KE) is $230 \mathrm{~m} \Omega$.

The power loss in the coupled inductors is:

$P_{L}=I^{2} R_{\text {leak }}=1,4^{2} \cdot 0,230=0,45 \mathrm{~W}$

The input inductor, by Coilcraft (MSS1260T-104ML) dissipates a power given by:
$P_{\text {Lin }}=1,4^{2} \cdot 0,146=0,28 \mathrm{~W}$

Taking losses into account, the efficiency is:

$\eta=\frac{P_{o}}{P_{\text {Mos }}+P_{L}+P_{\text {Lin }}+P_{o}}=0,94 \rightarrow 94 \%$

The result obtained is of great interest but it should be noted that it represents an estimate of the first order, since voltage and currents depend on the duty cycle which in turn depends on the overall efficiency. A more detailed analysis will not be conducted because evaluating efficiency is not the final aim of this work, the value achieved is already a very satisfying result.

\section{Modified scheme for direction indicators light}

A further typical application concerns the directional arrows, whose intermittent nature lends itself well to the effects of movement of light if the luminous elements are arranged in a continuous string of single elements.

LEDs are point sources, small in size and can be used to construct strings of any length, with or without passive optical elements and with a variable number of devices. Each LED can be controlled, in this way its activation is independent of whether other LEDs are switched on or off and dynamic lighting effects can be created.

The connection of the devices can be series or parallel based on the converter used and the control technique implemented. In a parallel configuration, the voltage must remain constant regardless of the number of LEDs. The converter must be able to supply the required current which increases each time a single LED lights up. In a series configuration, however, the current flowing through the LEDs is the same, changing the output voltage of the converter, which must be designed to adapt to these changes.

The designed converter, described in the previous section, does not come explicitly for this application but during the simulation it showed a good versatility, it was able to manage a sequence of LEDs in the correct way, with the addition of simple electronic components.

The implemented circuit diagram (figure 9) does not significantly differ from that proposed, except for some changes in the inductance values, in order to refine the performance for the new application, whose load is variable.

This is the main difference between what has been done and the need to supply a string in which every single ignition modifies the load seen by the converter. Variations in the load also affect the ripple, so the worst case must be taken into account to ensure uniform flow on the single LED.

The luminous effect imposes the following circuit dynamics: at the initial instant only the LED1 is 


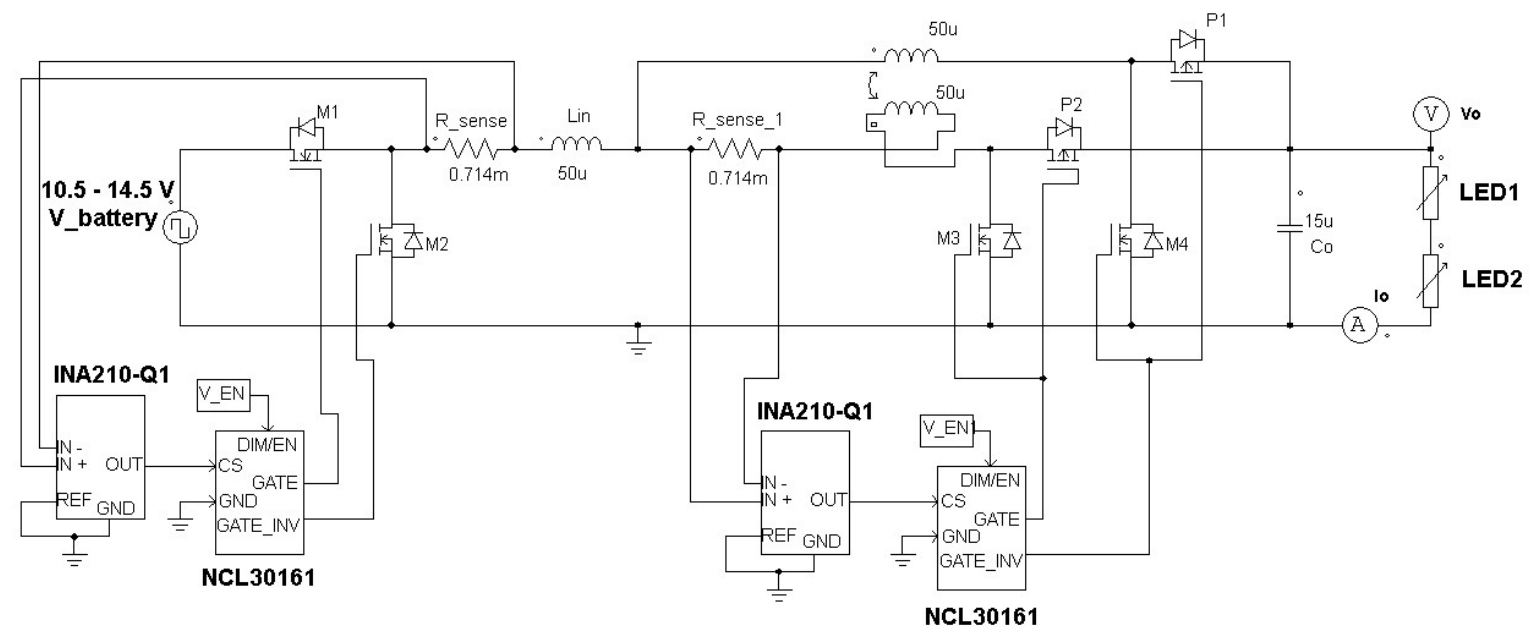

Fig. 8: commercial designed converter

supplied by the converter and the current flows entirely through it; after a precise time interval, LED2 must light up and LED1 must remain on, without the current changing, otherwise, the intensity of both will change. In short, after each time interval the next LED lights up and the others remain on; once the sequence is over, all of them are turned off and the cycle starts again.

To implement this condition, each LED is connected to the voltage reference by a switch. If the first LED in the series is to be driven, the corresponding switch is activated.

To turn on the second LED keeping the first on, just open the first switch and at the same time close the second one, and so on for the whole chain.

The time reference is imposed by a square wave that controls the first LED, when a low value is reached a monostable is activated which closes the next switch for a pre-established time and the same for all the others. The output of each monostable is connected to the input of the next, which is activated on the falling edge. This simple realization implements the operation described above.

\section{Results}

All the simulations were conducted on Powersimtech PSIM software.

The last aim of the simulation process is to verify that the LED is supplied with a constant current in any condition, with a ripple of $40 \mathrm{~mA}$ or less and with a transient behavior without any overshoot.

The output voltage for which the circuit has been sized is $20.4 \mathrm{~V}$ and the bias current $0.7 \mathrm{~A}$, these are the values that must result from the simulations carried out. Slight deviations from the expected values are acceptable and can not be traced back to an incorrect design but to approximations that are typical of the software and of the imposed sampling time. Some variations may also be caused by the values used as current thresholds, for example, and the imperfect ideality of the waveforms coming out of the comparators or voltage generators.

In the first analysis, the correct operation of the system is evaluated with a constant input voltage of $12 \mathrm{~V}$, equal to the nominal voltage of a common lead-acid battery for motor vehicles. The output current is shown in figure 10.

The average current value measured by the software is $0.695 \mathrm{~A}$, as expected. In the transient phase the current comes to the nominal value without any peak. The result obtained is excellent, it meets the target imposed by the application thanks to an effective control on the current of the first and second stage, as widely discussed above.

However, when turned on, for a few moments, the current is negative and reaches a value of $-0.65 \mathrm{~A}$. This behavior derives from the mathematical model the LED has been approximated to. As a math approximation, it is not reflected on the real operation of the device, which, as will be clear from the graph of the output voltage, will be subject to a positive voltage, without being in any way reverse-biased.

Figure 10 also shows the current ripple, whose peak-topeak value, although slightly variable over time, is limited from 0.68 to $0.71 \mathrm{~A}$.

As already mentioned, the calculation of the expected ripple was not carried out analytically, it was decided to set a value to be achieved and to act on the passive components.

To approximate the variable behavior of the supply voltage, a square amplitude of a very low frequency wave is used, it simulates slow variations in the voltage itself from 10.5 to $14.5 \mathrm{~V}$ (figure 11).

The average current value remains constant with a rather considerable input variation, denoting an excellent stability of the converter against changes in input voltage.

During the time in which the voltage reaches the minimum value, both the waveform and the ripple slightly change, this can be explained because the software works in discrete time, sampling the waveforms with an adjustable pitch and it has difficult to perfectly follow, during the transient, the sampled quantities. To confirm this last hypothesis, the simulations made occur, which show changes in the waveforms when the sampling time increases or decreases. 


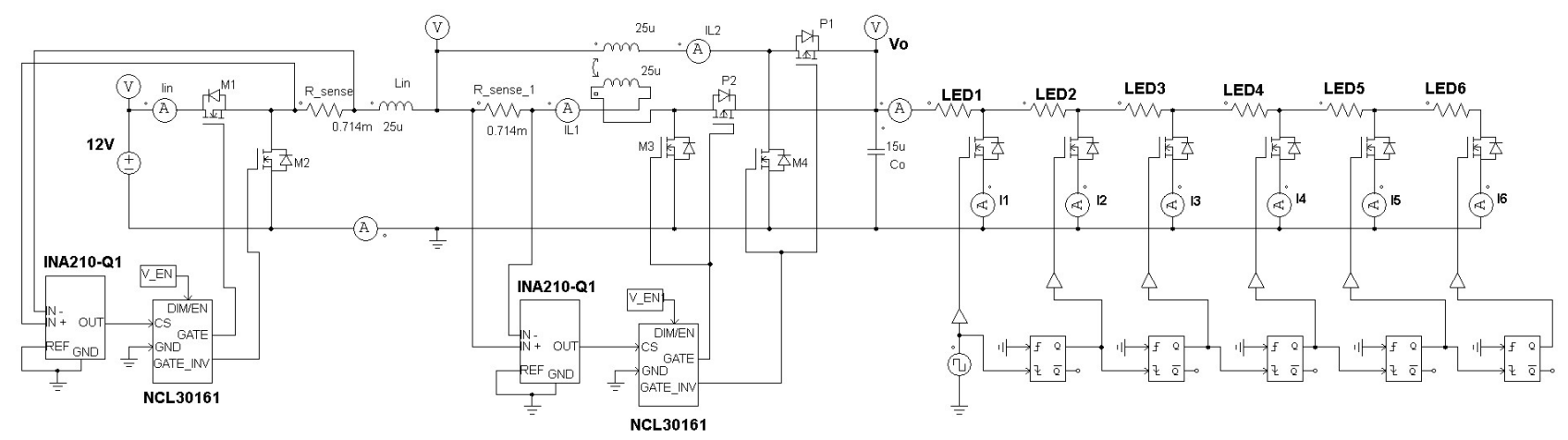

Fig. 9: electric scheme of the converter with a string of LEDs

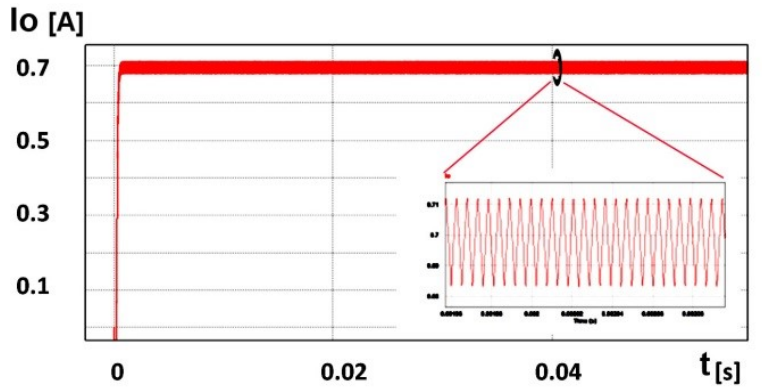

Fig. 10: output current (with zoomed ripple)

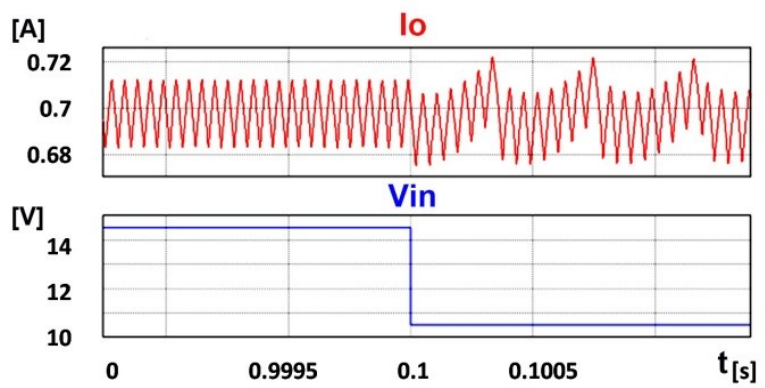

Fig. 11: output current vs input voltage

The designed converter can be also used in the control of directional arrows or in stop light, where a quick response of light to the command given by the driver or the interposed electronics is required. The luminous flux must reach the maximum in a very few instants and then return to zero at the same time.

In the realized converter, getting a discontinuous operation is very simple, just applying a PWM signal, at the desired frequency, to the current input of the current controller. The logic zero status of this signal turns off both the Boost stage transistor and the Buck stage input transistor and then disconnects the power supply, bringing the output current to zero. A high logic value, on the other hand, leaves the operation of the converter unaltered. The frequency determines the time an arrow is turned off/on.

The general behavior that can be obtained with the implemented scheme is visible in figure 12. The current has very squared edges, it rises and decreases almost like a square wave, this means that the transient is negligible compared to the times when the LED is off-on. Figure 13 shows the trend of the currents in the device for dynamic LED lighting.

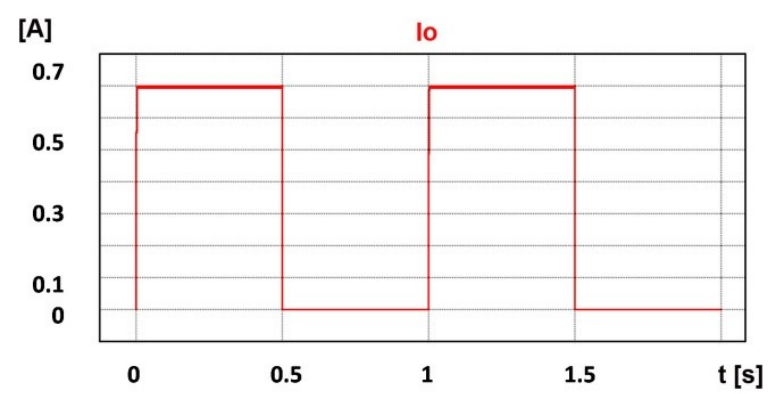

Fig. 12: output current for directional arrows

The current respects the predictions and is equal to 0.7 A. The fact that the current after a certain time goes to zero does not imply that the LED turns off, because the measurement is made on the switch and the switch must be opened to allow the previous LED to remain powered. To further clarify this concept, operation with only two LEDs can be considered. Both the voltage that drives the first switch, the voltage of the second switch, the overall behavior is shown in figure 14. By extension, whatever the LEDs are on, the current that supplies them is always the same, so the luminous flux remains constant during the entire ignition sequence.

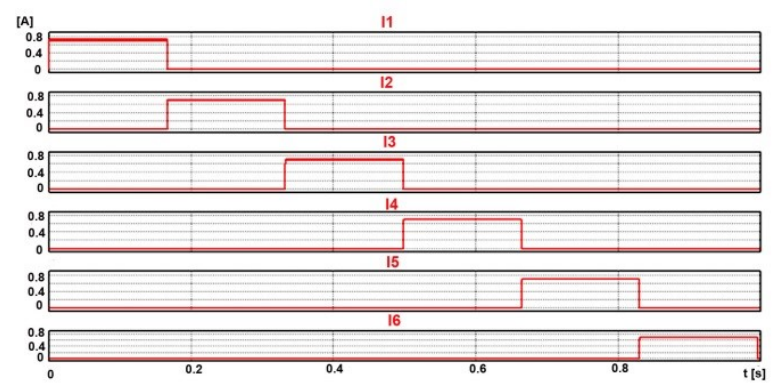

Fig. 13: temporal sequence of currents

The graph summarizing the trend of the current is shown in figure 15. The average current value is approximately constant, what changes is the ripple that is reduced with the increase in the number of supplied LEDs. However, it can be noted that in the worst case, i.e. with a single LED on, the ripple is still within the manufacturer's specifications.

However, it can be noted that in the worst case, i.e. with a single LED turned on, the ripple is still within the manufacturer's specifications. 


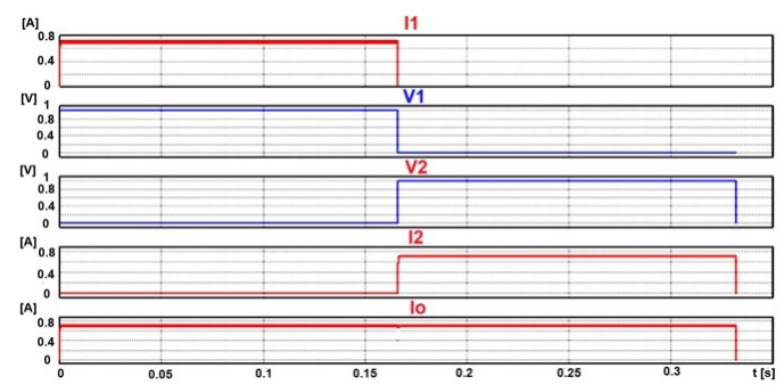

Fig. 14: implemented logic with two LEDs

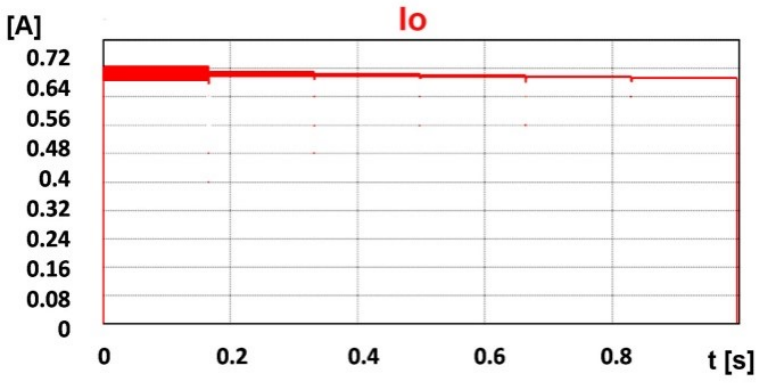

Fig. 15: total current behaviour while LEDs start up sequentially

\section{Conclusions}

A DC/DC converter, designed for LED lamps to be employed in vehicles both for lighting and directional arrows, has been proposed in this paper.

The circuit is composed of two stages. The input stages is a Buck converter whereas the output stages is a Boost interleaved,

The novelty consists on the use of a reduced number and size of passive devices, as a matter of fact, the circuit lacks both of the output bulk capacitor of the Buck stage and of the continuous filtering capacitor in the interleaved stage which is controlled as a stacked Boost. The control circuitry of both stages is designed by commercial integrated devices as well. The unique design constraint is on the intermediate voltage, which must be about half of the desired output voltage. Moreover, a small coupled inductor guarantees a reduced output ripple.

The DC/DC converter is able to be supplied by a wide range of $\mathrm{DC}$ voltage and gives a constant output current suitable for the LEDs.

The LED device has been experimentally characterized before implementing in the simulator.

Among the other advantages of the proposed solution, there are the use of low-cost commercial devices, able to be integrated into a chip or by SMD devices, a high reliability due to the simple design and to the presence of thermal and overcurrent protection circuits.

\section{References}

[1] Y. Sun, X. Wu, "High efficiency LED driver featuring auto output-voltage tuning", IEEE International Conference of Electron Devices and Solid-State Circuits (2010).

[2] S. Marconi, G. Spiazzi, A. Bevilacqua, M. Galvano, S. Stoegner, "Efficiency improvement in Automotive Rear Lighting applications: a practical industry-ready implementation", 50 ${ }^{\text {th }}$ Annual Meeting of the Associazione Società Italiana di Elettronica (SIE) Napoli, Italy, June 20-22, (2018).

[3] Y. Dubois, T. Krzesaj, "Automotive Lighting Facing the Design Trend: From Former Basic Conception to Reliable and Sustainable Advanced Development", electronic goes green conference, Berlin, Germany, 6-9 Sept. (2016).

[4] "Automotive lighting: technology, industry and market trends",Yole developement, Market and Technology report, https://www.slideshare.net/Yole Developpement/automotive -lighting-technology-industry-and-market-trends-2017report-by-yole-developpement (2017)

[5] "Lighting Systems: from light to advanced vision technologies",Valeo,https://www.slideshare.net/ValeoService /valeo-lighting-systems-from-light-to-advanced-visiontechnologies-valeoscope-technical-handbook-998542

[6] G. Vitale, "Energy saving by power electronics: towards a new concept of renewable source", International Conference on Renewable Energies and Power Quality (ICREPQ'16), Madrid (Spain) 4-6 May 2016,

[7] P. Giannelli, L. Capineri, G. Calabrese, G. Frattini, M. Granato "A reduced output ripple step-up DC-DC converter for automotive LED lighting", 13th Conference on Ph.D. Research in Microelectronics and Electronics, PRIME, Giardini Naxos, Italy, 12-15 June (2017).

[8] Y. Qu, W. Shu, J. S. Chang, "A Low-EMI HighReliability PWM-Based Dual-Phase LED Driver for Automotive Lighting" IEEE Journal of Emerging and Selected Topics in Power Electronics, Vol. 6, Issue: 3, pp 1179-1189, (2018).

[9] "Average Current Mode Control of Switching Power Supplies", Lloyd Dixon, Unitrode application note.

[10] N. Selvaraju, P. Shanmugham, S. Somkun, "Two-Phase Interleaved Boost Converter Using Coupled Inductor for Fuel Cell Applications", International Conference on Alternative Energy in Developing Countries and Emerging Economies AEDCEE, 25-26 May, Bangkok, Thailand (2017).

[11] H. Choi, "Practical Feedback Loop Design Considerations for Switched Mode Power Supplies", Fairchild Semiconductor Power Seminar (2010 - 2011).

[12] R. Lin, "Modeling and Compensator Design of LED Driver Systems", IEEE Industry Applications Society Annual Meeting, 1-5 Oct. (2017).

[13] Jose T.A., Joseph K.D "LED Modelling and Input Current Ripple Reduction of Cuk Converter using Magnetic Coupling", International Conference on Next Generation Intelligent Systems, Kottayam, India, 1-3 Sept. 2016 (2016).

\section{Acknowledgement}

The authors would like to thank prof. Mauro Mosca for the technical support given by the laboratory "Thin Films" at the Dipartimento di Energia, Ingegneria dell'Informazione e Modelli Matematici, Università di Palermo, in which the experimental characterization of the LED has been carried out. 